\title{
LAS AMBIGÜEDADES DE NUESTRA CULTURA TECNOCIENTÍFICA Y LA EDUCACIÓN. ALGUNAS REFLEXIONES
}

\author{
Agustí NIETO-GALÁN \\ Departament de Filosofia. Centre d'Estudis d'Historia de les Ciències. \\ Universitat Autonoma de Barcelona
}

\section{Introducción}

En la reciente obre The age of extremes, ${ }^{1}$ en la que el historiador británico Eric Hobsbawm hace un recorrido general por el siglo XX desde 1914 a 1991, se incluye un capítulo dedicado especialmente al papel de la ciencia y la tecnologia en las últimas décadas de nuestra historia ${ }^{2}$. Éste es ya un hecho significativo, que corrobora la sensación ampliamente compartida de que el siglo $\mathrm{XX}$ no se puede explicar sin la historia de su ciencia y su tecnologia, y que los tradicionales capítulos de dedicados a la economía, la política, la sociedad o la cultura en general, parecen insuficientes a la hora de hacer un retrato preciso de nuestra más próxima contemporaneidad.

En la versión original de Hobsbawn, el capítulo 18 se titula: «Sorcerers and Apprentices-The Natural Sciences", y se inicia con un fragmento de una entrevista realizada en 1988 a Claude Lévi-Straus, padre del estructuralismo y uno de los grandes intelectuales del siglo. En concreto, a la pregunta: ¿Usted cree que hay lugar para la filosofia en el mundo actual? respondía en los términos siguientes: "Evidentemente, pero sólo si está basada en los actuales conocimientos y descubrimientos científicos. Los filósofos ya no pueden luchar solos contra la ciencia. Esta ha transformado radicalmente nuestra visión de la vida y del universo, $\mathrm{e}$ incluso ha revolucionado las leyes que rigen nuestra inteligencia» ${ }^{3}$.

HobsbaWM, E. Age of Extremes. The Short 20th Century 1914-1991. London 1994.

Cap. 18: «Sorcerers and Apprentices-The Natural Sciences», págs. 522-557.

HobSBAwM, E. Age of Extremes...op. cit., pág. 522.

ENDOXA: Series Filosdficas, n. ${ }^{\circ} 14,2001, p p .321-339$. UNED, Madrid 
En la respuesta de Lévi-Straus sobre el estatus actual de la filosoffa se asume implícitamente que incluso nuestra manera de pensar, seamos científicos o no, está actualmente mediatizada por una nueva sociedad tecnocientífica que ha roto con tradiciones intelectuales anteriores, que ignora otros modelos culturales y que impone su visión hegemónica del mundo y su transformación. De hecho, parece tratarse de una especie de triunfo, que en otros tiempos podríamos llamar positivista, donde el necesario equilibrio entre la cultura humanistica y la cientifica, soñado por C.P. Snow en los años 1950 , pero nunca conseguido, parece haberse desplazado irreversiblemente hacia la hegemonía cultural del complejo científico y tecnológico de la segunda mitad del siglo $\mathrm{XX}^{4}$.

¿En que consiste esta supuesta victoria cientifica de la que hablaba LéviStrauss? ¿Es realmente irreversible, o debemos recuperar aspectos relevantes de la cultura humanística, aunque sea excavando elementos de su pasado? Quizás en el fondo se trata de construir una nueva balanza, con un nuevo plato que represente una cultura humanística renovada, capaz de reconducir o repensar parte de la nueva cultura científica hegemónica. Evidentemente, en función de las respuestas a estas preguntas, los modelos educativos deben también ser repensados. Según nuestra propia concepción del peso cualitativo y cuantitativo de la ciencia y de la tecnología en nuestra cultura, las formas y contenidos de su transmisión a los no expertos (prácticas entre las que evidentemente se encuentra la educación) diferirán de manera notable ${ }^{5}$. Quizás la exploración de algunos de los aspectos principales de nuestra cultura tecnocientifica nos puede permitir encontrar nuevas claves educativas.

\section{El fin de la filosofía natural y la ciencia «moderna»}

Hasta ya bien entrado el siglo XIX, los interesados en explicaciones racionales sobre el funcionamiento de la naturaleza eran conocidos como filósofos

4 SNOw. C.P. Les dues cultures i la revolució cientifica. Laie. Barcelona 1965. (1a. ed. en inglés, 1959); DAVENPORT, W.H. Una sola cultura. La formación de tecnologos-bumanistas. Barcelona 1979. (1a. ed. en inglés, 1970). Capítulo 1: "Las dos culturas: otro vistazo a imágenes y actitudes" págs. 13-36.

5 En relación al problema de la cienia y su trasmisión a diversos públicos, ver, por ejemplo: Shapin, Steven, "Science and the public" a OLbY, R.C., G.N. CANTOR, J.R.R. ChRISTIE, M.J.S. HoDGE (eds.) Companion of the History of Modern Science. Routledge. Londres 1990. págs. 990-1007. 
naturales, entre los que podríamos contar a los grandes protagonistas de la revolución científica de los siglos XVI y XVII: Galileo, Copérnico, Descartes, Bacon, o el mismo Newton. Aquella filosofía natural, que heredaba la tradición intelectual de las Universidades medievales, integraba elementos teológicos y médicos, con una presencia creciente de matemáticas, historia natural, óptica o astronomía, a veces influidas por algunos elementos mágicos o alquímicos, que todavía hoy provocan discusiones en relación a su papel como impulsores o retardadores del desarrollo de la llamada ciencia moderna ${ }^{6}$. A menudo los historiadores de la ciencia han visto en la revolución científica de los siglos XVI y XVII, con su división baconiana del saber y la aparición de la ciencia experimental, una primera fractura significativa de la vieja filosofía natural y han usado esa categoría histórica usada como un punto de referencia legitimador del modelo de ciencia actual ${ }^{7}$. Probablemente no se puede negar que en este largo proceso se ha ganado eficacia, especialización, capacidad de transformación de la naturaleza a través de grandes cambios tecnológicos, pero también se ha perdido perspectiva global. Se conocen muy bien desde el punto de vista operativo algunas piezas del engranaje, pero la reflexión sobre las características generales de la máquina se ha empobrecido.

En cualquier caso, el siglo XX ha sido sin duda el siglo de la ciencia ${ }^{8}$. En 1910 había en toda Europa Occidental unos 100.000 científicos mientras que en 1980 hemos llegado a cinco millones de investigadores, de los cuáles aproximadamente un millón está en los Estados Unidos ${ }^{9}$. El número de artículos publicados se dobla cada 10 años, y la progresión de crecimiento puede llegar

6 Las revisiones historiográficas más recientes de la llamada "Revolución cientifica" incluyen elementos de la magia, la alquimia o el hermetismo para comprender la introducción de la filisofia natural experimenrtal. Ver por ejemplo, HENRY, J. "Magic and Science in the 16th and 17th Centuries" a OlBY, R.C., G.N. CANTOR, J.R.R. ChRISTIE, M.J.S. HodGe (eds.) Companion of the History of Modern Science. págs. 583-596; SCHUSTER, J.A. "The Scientific Revolution" a Olby, R.C., G.N. CANTOR, J.R.R. CHRISTIE, M.J.S. Hodge (eds.) Companion of the History of Modern Science...op. cit., págs. 217-242; LINBERG, D.C., WESTMAN, R.S. (eds.) Reappraisals of the scientific revolution. CUP. Cambridge 1990.

Esta podría ser la tesis tradicional de BuTTERFIELD, Herbert, Los origenes de la Ciencia Moderna. Taurus. Madrid 1982. (1a. ed. inglesa, 1949). Capítulo X: "El lugar que ocupa la revolución científica en la historia de la civilización occidental" págs. 177-192.

8 Para una panorámica global de los grandes logros de la ciencia del siglo XX, ver: KRIGE, John and PeSTRE, Dominique, "Introduction" a KRIGE, John and PESTRE, Dominique (eds.) Science in the Twentieth Century. Harwood. Amsterdam 1997.

9 HoBSBAWM, E. Age of Extremes...op. cit., págs. 522-523. 
a ser realmente astronómica en el siglo XXI. Se ha creado una comunidad científica internacional controlada per unos pocos países desarrollados que marca los estándares y criterios de calidad e interés de la investigación. El atractivo de la internacionalización del hecho científico tiene como contrapartida el triunfo de la homogeneidad de unos pocos frente a la heterogeneidad de los muchos. La ciencia se ha convertido también en un instrumento de dominio cultural, tecnológico, estratégico o militar, y las reglas de su juego parecen cada vez más rígidas. La pequeña República de las ciencias constituida por los filósofos naturales de la Europa de los siglos XVII y XVIII ${ }^{10}$, más próxima a una reunión de aristócratas inquietos o refinados bufones de monarcas mecenas se ha transformado en una máquina sofisticada y compleja donde la individualidad, la creatividad o la particularidad tienen un espacio muy reducido.

En cualquier caso, ésta es probablemente otra de las paradojas de la historia. Los siglos XVIII y XIX están llenos de nuevas sociedades científicas, de luchas encarnizadas por institucionalizar nuevas disciplinas como la química, la geología, o la biología, por organizar nuevas comunidades de ingenieros, naturalistas, médicos o cirujanos ". Eran también tiempos de crecientes vínculos con el mundo industrial a su vez en franca expansión, y de grandes esfuerzos de difusión de los nuevos valores científicos y de múltiples intentos de convencer a los poderes políticos y a las audiencias ilustradas de la conveniencia de promocionar aquellas actividades, que hablan comenzado como diversiones intelectuales, como curiosidades innatas, y que a través de la enseñanza técnica se pueden ahora convertir incluso en instrumentos de control social para apaciguar las tensiones generadas por las masas obreras de Manchester o Liverpool en la Inglaterra Victoriana ${ }^{12}$.

En 1800, Alessandro Volta presentaba al emperador Napoleón su famosa pila electroquímica que generaba electricidad a través de un proceso de oxidación y

10 GaY, Peter, The Enlightenment. An Interpretation. (2 vols.) W.W. Norton and Co. New York, London 1977 (1a. ed. 1966)., II, 56-83.

11 Para el problema de la profesionalización de los científicos, ver trabajos clásicos como: BEN-DAVD, J. Scientific Growth. Essays on the Social Organization and Ethos of Science. Berkeley: Univ. of California Press, 1991; KNIGHT, David. La era de la Ciencia. Ed. Pirámide.Madrid 1988 ( 1 a ed. en inglés 1966). Para revisiones constructivistas del problema de la profesionalización, ver: GolinSK, Jan, Making Natural Knowledge. Constructivism and the History of Science. Cambridge University Press. Cambridge 1999.

12 Fox, Robert, GuAGnini, Anna (eds.) Education, Technology and Industrial performance in Europe, 1850-1939. CUP Cambridge 1993. 
reducción de metales. Bonaparte se habla rodeado de eminentes científicos para ocupar cargos políticos de alta responsabilidad durante los primeros años del siglo XIX. Michael Faraday realizó espectaculares experimentos de física y química en un teatro lleno de inquietos "gentlemen" en la "Royal Institution" de Londres, y los nuevos químicos, discípulos del gran Justus von Liebig en la Universidad de Giessen, en Condado de Hessen, se integraban en las industrias químicas que transformaron el papel de la química desde el punto de vista tecnológico en la segunda mitad del siglo XIX ${ }^{13}$.

La ciencia ha ganado capacidad de influencia política, económica y social, y la lucha continuada de los nuevos filósofos naturales del 1800, los que progresivamente se reconocerán como científicos para conseguir un nuevo espacio intelectual, institucional, profesional y público, se ha convertido en nuestra época ya una condición evidente. El científico se instala hoy en sólidas y complejas estructuras de investigación y enseñanza que han adquirido un elevadísimo grado de organización y tecnificación, y que al mismo tiempo que le facilitan su trabajo le obligan a adquirir un lenguaje y unos conocimientos altamente especializados si no quiere ahogarse en un mercado laboral cada vez más exigente y precario.

Durante el siglo XIX las ciencias naturales se incorporaron a los planes de estudios de universidades y escuelas técnicas, y las relaciones personales entre maestro y alumno de carácter gremial y artesanal, que por ejemplo se daban en los "Cabinets de Chimie et Physique" de la Francia del siglo XVIII desaparecieron progresivamente. Además, en los ańos 1830 y 1840 nacieron los llamados grupos de investigación o "research shcools", una nueva manera de ensefiar y de hacer investigación en equipo, con una estructura organizada y con una creciente división del trabajo que facilitaba la incorporación de nuevos doctorandos a las líneas de investigación del grupo ${ }^{14}$. En realidad, la estructura de aquellos primeros grupos de investigación no es muy diferente de la de muchos laboratorios universitarios de la actualidad, pero la complejidad técnica de aparatos, medidas, estándares, y la proliferación de empresas de gran envergadura, la llamada "big science", han revolucionado la sociología de la investigación y la enseñanza científica, hasta el punto de que algunos estudiosos de la política cientifica actual, como el profesor inglés Michael Gibbons,

13 KNIGHT, David. La era de la Ciencia...op. cit.

14 GeIsON, G., HOLMES, F.L. (eds.) Research Schools. Historical reappraisals, Osiris, 8, 1993. 
opinan, que en el siglo XXI la estructura de departamentos universitarios será totalmente obsoleta y los nuevos proyectos de investigación tenderán a ser sofisticadas inversiones temporales en recursos humanos y tecnología totalmente reconvertibles una vez conseguido un objetivo concreto al cabo de tres o cuatro años, tal com se está desarrollando el proyecto del genoma humano. Un ejemplo significativo de esta "gran ciencia" de inversiones millonarias y de proporciones tecnológicas y humanes enormes es el acelerador de partículas LEP (Large Electron Positron Collider) de $27 \mathrm{Km}$ de perímetro en el Laboratorio Europeo de Física de partículas (CERN) de Ginebra, donde científicos y técnicos de primera línea son contratados para alimentar el santuario de la ciencia moderna, que combina la búsqueda de nuevas partículas elementales con un amplio espectro de aplicaciones concretas de su investigación básica ${ }^{15}$.

En ese espectacular proceso de crecimiento desde el siglo XVIII hasta nuestro presente la ciencia ha experimentado un proceso de profunda especialización, profesionalización, organización y tecnificación, y todo investigador necesita hoy, para desarrollar una nueva línea de investigación, estar acompanado de esta tupida red de servicios, máquinas y recursos humanos. La vieja tradición de la filosofía natural se ha transformado radicalmente, sin duda, a causa de la profunda especialización y el papel creciente de la tecnologia. Dos factores que me gustaría desarrollar con un cierto detalle.

\section{El científico profesional y la especialización}

De hecho, las subdivisiones del saber desde del punto de vista intelectual e institucional, han variado continuamente durante toda la historia de la ciencia, y todavía hoy somos testimonios de las continuas discusiones académicas para adecuar los cirrículums docentes y investigadores a nuevas necesidades de una sociedad en acelerado cambio. Las viejas medicina, teología, leyes como pilares básicos del saber medieval se han transformado hoy quizás en genética, electrónica y economía, transgrediendo incluso las ya clásicas ciencias naturales (física, química o biologia) que luchaban por un espacio nuevo en el siglo XIX. En los años 1960, Hobsbawm era «Researh fellow» del King's College de la Universidad de Cambridge, en la misma época en que James Watson y

15 KrIGE, John and PeSTRE, Dominique (eds.) Science in the Twentieth Century...op. cit. 
Francis Crick trabajaban en la determinación de la estructura química del famoso $\mathrm{ADN}$, que había de revolucionar posteriormente la genética y la biología molecular. A menudo, se encontraban después del trabajo en el mismo pub bebiendo cerveza, pero con el convencimiento de que el autor de The Age of Revolution. Europe 1789-1848, y de The Age of Capital 1848-1875 (dos de las obras clave de Hobsbawn y un trabajo excelente de la escuela marxista inglesa de los años 60) ${ }^{16}$, nunca entendería la revolución de la doble hélice del $\mathrm{ADN}$. Un sentimiento de lejanía probablemente superior, por ejemplo, a la posible distancia cultural entre la revolución química de Lavoisier a finales del siglo XVIII y la toma de la Bastilla, un hecho emblemático de la primera revolución burguesa de 1789 .

C. Petera Snow, un químico que estudiaba la estructura de la vitamina A en los laboratorios de Cambridge, intentó establecer un puente de diálogo entre cientificos y humanistas cuando escribió en 1959 un famoso libro, antes citado, que recogía algunas de sus conferencias de los años precedentes titulado Las dos culturas $y$ la revolución cientifica, donde denunciaba la fractura irreversible que se había producido en la cultura occidental entre la tradición humanista, que recogía alguno de los valores y métodos de aquel filósofo que Lévi Strauss consideraba ya superado, y la tradición científica que después de tertulias elitistas, universidades, industrias, departamentos, máquinas y computadores, había generado un lenguaje extremadamente particular y inasequible, a pesar de los esfuerzos de divulgación.

Snow, de hecho, recogia ya algunas críticas que personalidades remarcables habían hecho durante las primeras décadas del siglo XX sobre la hegemonía cultural de la ciencia en Occidente y, progresivamente en todo el mundo. Bertrand Russell había denunciado el peligro de un posible despotismo de la ciencia; Georges Sarton, uno de los fundadores de la historia de la ciencia como disciplina académica en los Estados Unidos después de la Segunda Guerra Mundial, reclamaba la necesidad de desarrollar un humanismo cientifico; el historiador de la ciencia china Joseph Needham se manifestaba ya en 1925 contra la especialización y división del conocimiento en departamentos y contribuía a una tradición, enfatizada por Snow, sobre la necesidad de construir nuevos puentes de comunicación entre diferentes áreas del saber. Además, las guerras mundiales y la cultura hippie de los 1960 s contribuyeron a denunciar

16 Existen numerosas ediciones de la traducción al castellano de las dos obras de Hobsbawn. 
el abuso material e intelectual de la cultura científica, a reconsiderar desde un punto de vista contracultural la utilidad de aquella vieja idea ilustrada del progreso o a reconducirla hacia el progreso que en teoría representaba la justicia social del mundo comunista, una idea que influyó considerablemente en el profesor de cristalografía de Cambridge Joseph Bernal, quien en 1964, publicó la versión definitiva de su Historia social de la ciencia ${ }^{17}$.

En el fondo, Snow, convivia en su College de Cambridge con estudiantes y investigadores de todos los ámbitos del saber, uno de los aspectos originales y más interesantes del sistema universitario inglés de Oxford-Cambridge, un mundo elitista y aristocrático que ya durante el siglo XIX se resistió duramente a la introducción de las nuevas ciencias naturales en los currículums educativos impregnados de la vieja tradición de la educación liberal inglesa, donde la cultura clásica greco-latina, junto con una visión holística, literaria y antiutilitarista del saber representaban según la tradición el más adecuado para la formación integral del individuo, que, sin duda se empobrecía intelectual y humanamente, y se lo sometía a una especialización excesiva, demasiado rígida y matematizada. Ese estilo particular del sistema Oxbridge preservaba, y quizás todavía mantiene actualmente, al menos en las disciplinas humanísticas, algunos de los valores que parece imprimía un carácter especial a los graduados de esas universidades, los cuáles ocupan la mayoría de los cargos públicos, técnicos y políticos de la Gran Bretaña.

En el contexto de la universidad de Berlín, la distinción establecida a principios del siglo XIX por Wilhelm von Humboldt entre Wissenschaft (conocimiento abstracto libre de su aplicación inmediata) y Bildung (educación moral e intelectual del individuo) ponía las bases institucionales par el futuro desarrollo de la investigación básica, como un valor intelectual prioritario, con la intención de liberar a la ciencia de una excesiva dependencia de proyectos utilitarios aplicados a las necesidades industriales a corto plazo. Institufa además el seminario de investigación como uno de los pilares de las research schools germanas capitaneadas por Justus von Liebig en Giessen. Además, las nuevas Facultades de Ciencias napoleónicas concedían al científico profesor universitario un estatus de funcionario servidor del nuevo estado ${ }^{18}$.

17 Rossi, Paolo, Las arañas y las hormigas. Una apologia de la historia de la ciencia. Crítica. Barcelona 1990. (1a. edición italiana 1986).

18 FoX, Robert, WEISZ, Georg (eds.) The Organization of Science and Technology in France 1808-1914. Cambridge University Press. Cambridge 1980. 
La necesidad de compartimentación del saber a menudo por razones prácticas y perfectamente justificables ha producido una profunda fractura en el conocimiento humano de finales del siglo XX, ha traicionado el sabio del Renacimiento, el espíritu enciclopédico del siglo XVIII y ha superado dramáticamente las viejas divisiones baconianas para generar expertos que saben casi todo de casi nada. El especialista, además, ha contribuido a desplazar las aproximaciones globalizadoras y las grandes preguntas del filósofo sobre el por qué y el cómo del mundo que nos rodea, y ha marcado unos límites operativos y prácticos para la actividad cotidiana de investigación y aplicación técnica destinada a resolver un problema concreto, que por otro lado puede tener una "gran" utilidad social, como a menudo algún tipo de divulgación científica acrítica se encarga de propagar. Snow tenia razón cuando reclamaba la construcción de nuevos puentes de diálogo interdisciplinar, que ahora deberían hacerse extensivos a la comunicación entre las mismas disciplinas científicas.

Nada más lejos de mi propósito que reivindicar un retorno nostálgico a la vieja filosofía natural. La irreversibilidad del tiempo histórico y la necesaria aceptación (aunque crítica) al contexto cultural en el que vivimos, me hace sugerir, sin embargo, la necesidad de corregir tendencias negativas como la de la excesiva compartimentación. Los puentes de Snow no son evidentemente sencillos de construir, pero sin, por ejemplo, un refuerzo y una reforma de las humanidades en los proyectos educativos, o sin una reflexión seria de lo que significa esta cultura científica para los habitantes del planeta a finales del siglo $\mathrm{XX}$, las aguas pueden bajar cada vez más turbias agrandando el abismo ya existente actualmente entre progreso científico y progreso social.

La emergencia de nuevos discursos transversales capaces de liberar a los alumnos de la rigidez disciplinaria tradicional han de combinarse con una profunda renovación del contenido científico y técnico de los planes de estudios a diferentes niveles, tal como ha preconizado en buena parte en los últimos años el movimiento "Ciencia-Técnica-Sociedad".

\section{El papel de la tecnología}

El segundo factor que quisiera considerar es el del acelerado cambio tecnológico que nos ha tocado vivir. De hecho, en los últimos doscientos años se han producido transformaciones radicales en los usos de la energía desde las primeras máquinas de vapor del siglo XVIII, la electricidad, o los motores de combustión 
interna. Esos ingenios fueron aplicados en un nuevo sistema industrial de organización creciente para la producción masiva de objetos de consumo que llenan la vida de los ciudadanos del primer mundo y que se extienden al resto del planeta en un espacie de intercambios económicos cada vez más estrechamente relacionados gracias a la revolución en los transportes y las comunicaciones que la misma tecnología ha proporcionado ${ }^{19}$.

El siglo XX ha nacido con automóviles, aviones, cine y radio mezclados con la relatividad, la mecánica cuántica y la genética, como fruto de una tendencia imparable durante el siglo XIX de la llamada "science based industry" donde el desarrollo industrial y tecnológico ha establecido vínculos estrechos con la llamada ciencia pura o básica creando nuevos vínculos y realimentaciones que hacen casi imposible establecer una relación causa-efecto y que han desdibujado aquella idea, demasiado simplista, de la técnica com un producto de la aplicación directa de la ciencia ${ }^{20}$. Ciencia y técnica no se explican una sin la otra, hasta el punto de que, por ejemplo, las evidencias de la existencia de una nueva partícula elemental (un quark) requieren de un sofisticadísimo sistema informático de detección que centra las discusiones de los investigadores en expertos que entienden y manipulan el sistema computerizado. Las inversiones en infraestructura técnica para desarrollar nuevas líneas de investigación son cada más elevadas e imprescindibles. En el fondo vivimos en un mundo de complicadas cajas negras que la mayoría de la población utiliza sin comprender com funcionan, y donde el diálogo entre especialistas de campos diferentes es cada vez más difícil, e imposible con los no científicos, que continúan siendo la mayoría de la población. Es curioso observar cómo filósofos, científicos, administrativos, artistas o parados pagan apresurados sus facturas en los supermercados modernos donde un trabajador de baja cualificación manipula sin problemas un misterioso sistema de códigos de barras que le permiten gestionar las salidas de los clientes.

Una de las grandes paradojas se encuentra precisamente en el hecho de que si bien esta tecnociencia ha obtenido resultados exitosos en la biotecnología, la

19 Para una panorámica general, en clave divulgativa, de estos cambios tecnológicos, ver: Buchanan, A.R. The power of the machine. The impact of Technology from 1700 to the Present. Viking. Londres 1992.

20 Para un estado de la cuestión de la historia de la tecnología, ver: Fox, Robert (ed.) Technological Change. Methods and Themes in the History of Technology. Harwood Academic Publishers. Amsterdam 1996. 
fisión nuclear, la superconducción, el láser, la medicina, la simulación o la informática, ha generado al mismo tiempo, a causa probablemente de algunos de los problemas que acabo de citar, un conjunto de incomodidades y reglas, que han convertido el siglo de la ciencia en también el siglo de la crisis de aquel viejo sueño positivista del XIX del progreso científico y técnico como vía segura e indiscutible hacia la felicidad humana ${ }^{21}$. Y así el siglo XX se ha convertido probablemente en el momento de más grave divorcio entre las dos culturas de Snow, donde esta nueva ciencia ha generado perplejidad por su discurso incomprensible, desconfianza por la incertidumbre de los efectos de su aplicación hasta las últimas consecuencias, e indefensión del individuo delante de la poderosa estructura de la «big science" que agrava la sensación de alejamiento entre el experto y el ciudadano medio.

De hecho, los suefios de los paraísos de la tecnología de Jules Verne o $\mathrm{H}$. George Wells a finales del siglo XIX (viajes espaciales, ciudades futuristas, o máquinas del tiempo) ${ }^{22}$. se han convertido hoy a menudo en pesadillas de residuos urbanos, accidentes nucleares, superpoblación, contaminación, extinción de especies, guerras químicas o agujeros en la capa de ozono ${ }^{23}$. En algunos momentos parecen renacer antiguos mitos sobre las limitaciones humanes en relación al reto de la comprensión y transformación de la naturaleza: Como Adán y Eva y el pecado original de la manzana del árbol de la ciencia en el Génesis bíblico; o Pandora, quien, según la mitología griega, inducida por una fatal curiosidad por abrir la caja que Zeus había confiado llena de todos los males que devastarían la tierra; o quizás Prometeo, portador del fuego, de la sabiduría de los humanos y de su capacidad de iniciativa, que Zeus castiga encadenándolo a una columna y enviándole un águila para devorarle lentamente el hígado; o Frankenstein, el personaje monstruoso de la novela de Mary Shelley de 1818, que condena la pretenciosa audacia de un excéntrico doctor que pretende erigirse en un nuevo Dios, amo de la vida y la muerte.

21 En relación a las actitudes ambigüas sobre la tecnologia, ver: BAUER, M. (ed.) Resistance to new technology. Cambridge University Press. Cambridge 1994.

22 ALKON, P.K. Science Fiction before 1900. Imagination discovers technology. Twayne Publishers. New York. 1994.

23 Para el análisis de los problemas medioambientales del desarrollo tecnológico, ver: WORSTER, Donald. Nature's Economy: $A$ History of Ecological Ideas. CUP. Cambridge 1985; WORSTER, Donald. The Ends of the Earth. Perspectives on Modern Environmental History. Cambridge University Press. Cambridge 1988. 
De hecho, un conjunto de debates en diferentes campos de la ciencia actual y sus aplicaciones se encuentran hoy en día más abiertos que nunca: los límites, si es que deben existir o no en las aplicaciones de la ingeniería genética, la definición científica de la muerte, la investigación militar, el sentido de la ciencia como un bien público, el impacto de los medios de comunicación electrónicos, o los criterios de prioridad en la investigación, entre otros, continúan arrastrándonos al dilema entre la necesidad de buscar policías supuestamente justos que vigilen los desmanes de Eva y Adán, Pandora, Prometeu, y Frankenstein, o contrariamente, confiar en sus arriesgadas iniciativas como único medio innegociable de superación humana ${ }^{24}$.

Es suficientemente conocido que el físico alemán Otto Hahn, emigrado a los Estados Unidos huyendo como muchos otros de la amenaza nazi, descubrió la fisión nuclear en 1939, un hecho clave que abría la posibilidad de construcción de la bomba atómica que acabaría aniquilando las poblaciones japonesas de Hiroshima y Nagasaki en $1945^{25}$. Pero, de hecho, Nils Bohr, el padre del modelo atómico que revolucionaría la física del siglo veinte, estaba convencido que el gran descubrimiento de Hahn sería del todo inútil desde el punto de vista de su aplicabilidad. Esta es una historia repetida, y que evidentemente descarga de responsabilidad de los cientificos respecto a algunos usos perversos de nuevas aportaciones básicas aparentemente inútiles. Quizás la ciencia, tal como han propuesto recientemente los sociólogos americanos Harry Collins y Trevor Pinch, no es ni buena ni mala, nos ofrece al mismo tiempo accidentes nucleares y remedios para enfermedades, accidentes mortales y autosufuciencia alimentaria potencial. Es com una especie de "Golem", una criatura amorfa de la antigua tradición hebraica que no es ni buena ni mala, poderosa pero potencialmente agresiva, un ser simpático que en un momento dado puede perder el control y causar un daño inmenso ${ }^{26}$.

Hablamos además de una historia de la tecnología cuya componente cultural la convierte en una disciplina integradora y enriquecedora del panorama historiográfico en renovación. Estamos ya lejos de los tiempos de las biografias hagiográficas de los genios inventores al estilo de Samuel Smiles, de las narraciones

\footnotetext{
24 KRIGE, John and PESTRE, Dominique (eds.) Science in the Twentieth Century...op. cit.

25 SANCHEZ-Ron, J.M., El poder de la ciencia. Alianza. Madrid 1992.

26 Coluins, Harry M, PINCH, Trevor, El goblem. Lo que todos deberiamos saber acerca de la ciencia. Crítica. Barcelona 1996.
} 
positivistas y teleológicas de los primeros ingenieros-historiadores, o de las historias acumulativas del progreso técnico al estilo de Maurice Daumas o Charles Singer ${ }^{27}$. En el fondo, ya en el espíritu de la revista Technology and Culture, fundada el 1958, Melvin Kranzberg, uno de los padres de la renovación historiográfica de la tecnología, veía muy clara la necesidad de integrar elementos técnicos en un discurso histórico más amplio. Ese contexto intelectual, que la SHOT y la prestigiosa revista Technology and Culture han conseguido desarrollar con un éxito notable en las últimas décadas, contribuye a reforzar la idea de que muy probablemente la historia de la tecnología cobrará un protagonismo muy grande en los próximos años, tal como el mismo Bernard Cohen auguraba en su seminario en Barcelona a finales del pasado año académico.

Melvin Kranzberg, el prestigioso fundador de la Society for the History of Technology americana (SHOT), definía sus propias leyes para la comprensión del fenómeno tecnológico desde la perspectiva histórica. La primera se formulaba en los términos: "la tecnología no es ni mala, ni buena, ni neutral". Es decir, la calificación ética de un hecho tecnológico determinado escapa al análisis histórico riguroso. Así, por ejemplo el famoso DDT que se presentó en Occidente como una panacea contra las plagas con el consiguiente progreso agrícola, acabó generando graves problemas sanitarios y medioambientales, tal como denunciaba Raquel Carson en su famoso Silent Spring de 1962. Pero, paradójicamente el mismo DDT se ha mostrado útil para la lucha contra la Malaria en la India y otros países en desarrollo. Además, Kranzberg presentaba una tecnología íntimamente ligada al hecho cultural, y definida con otras cinco leyes, en los siguientes términos: la invención es la madre de la necesidad; la tecnología se desarrolla en paquetes o sistemas; los factores no estrictamente técnicos tienen mucha importancia en los procesos de toma de decisiones técnicas; la historia de la tecnología es una pieza de gran importancia para la historia en general; la tecnología es una actividad esencialmente humana, como también lo es la historia de la tecnología ${ }^{28}$.

27 Daumas, Maurice, Histoire Générale des Techniques, 3 vols. PUF, Paris 1963. (traducción inglesa, Crown, Nueva York 1979-1980); Singer, Ch., HolmYard, E.J., Hall, A.R., Williams, J. A History of Technology. Oxford University Press. Oxford 1954-1958. (5 vols.)

28 KRANZBERG, Melvin, "Technology and History: 'Kranzberg's Laws" a REYNOLDS, T.S., CutCliffe, S.H., (eds.) Technology and the West. A Historical Anthology from Technology and Culture. The University of Chicago Press, Chicago 1997. págs. 5-20. 


\section{Otras ambigüedades de nuestra cultura científica}

La revolución experimentada per la fisica en los años entre las dos guerras mundiales representa quizás un momento donde un grupo importante de cientificos se han hecho preguntas profundas sobre el por qué y el cómo de la naturaleza. Los Einstein, Planck, Bohr, Sommerfeld, Pauling, Fermi, Dirac, y la nueva teoría de la relatividad y la mecánica cuántica abandonaban las explicaciones clásicas para entrar en un mundo de probabilidad, de complejos modelos matemáticos, sin menospreciar los intentos posteriores de encontrar una teoría unificadora unívoca que explicara el comportamiento de la materia, la energía, la electricidad o el magnetismo. Una "teoría del campo unificado" a la búsqueda de la belleza de la simplicidad de la naturaleza ${ }^{29}$.

Como señala Hobsbawn, ningún gobierno del mundo habría sonado antes de 1940 con la posibilidad de gastar una minúscula fracción de su presupuesto en proyectos especulativos de académicos excéntricos basados en cálculos incomprensibles ${ }^{30}$. Quizás ésta ha sido uno de las paradojas de la ciencia del siglo XX. Una parte del antiguo espíritu de la filosofia natural no ha muerto del todo. El descubrimiento continuado de partículas subatómicas y el crecimiento del nombre de quarks identificados en los últimos años mantienen vivo el interés por la compresión de aquello que en el siglo XVII los filosofos naturales habian denominado lo infinitamente pequeno y buscaban a través de los primeros microscopios, y la multiplicidad de características de estas subpartículas representan un reto para aquellos que buscan todavía aquella belleza de la naturaleza en su simplicidad.

La teoría del "big-bang" y los estudios sobre el origen y las características del universo nos acercan a aquel sueño de los que miraban a través del telescopio de Galileo para buscar todos los detalles de lo infinitamente grande que hay en el cielo y que se parecía más de lo esperado a la materia terrenal. Además, retornan a la investigación científica la dimensión de la explicación histórica de las cosas. La necesidad de pensar cual ha sido el proceso que nos ha llevado a una punto determinado de expansión de las galaxias de una manera análoga a como Jussieu, Cuvier, Lyell y los otros naturalistas del siglo XVIII comenzaron a imaginar el origen de la tierra a partir de las evidencias fósiles, o como el pensamiento evolucio-

29 SANChez-Ron, J.M., El poder de la ciencia....op. cit.

30 Cap. 18: "Sorcerers and Apprentices-The Natural Sciences"...op. cit. 
nista de Lamark o Darwin construyó una explicación histórica sobre la vida del planeta. La ciencia se aprendía habitualmente a partir de un discurso histórico a menudo construido por los mismos cientificos que aparecía en los primeros capitulos de los libros de texto de los siglos XVIII y XIX. Hoy el divorcio de las dos culturas ha eliminado prácticamente la aproximación histórica a la comprensión de las ciencias modernas cuando éstas son enseñadas a los futuros profesionales. Sólo algunos nombres anecdóticos permanecen a las lecciones introductorias antes de entrar en los detalles técnicos o científicos de cada disciplina.

Otros discursos intentan enriquecer la práctica cientifica recuperando algunas tradiciones anteriores y al mismo tiempo encontrar un punto de equilibrio en los usos y abusos de la tecnología. La teoría del caos generada durante los años 197080 parece que tiene algunos aspectos en común con la visión de la naturaleza romántica de la "Naturphilosophie» alemana de principios del siglo XIX. Dos de los pioneros de ese nuevo campo de investigación, Feigenbaum y Libchaber, parece que se inspiraron en la lectura de Goethe y en su critica a la teoría de los colores de Newton y en su tratado sobre la transformación de las plantes. En la naturaleza romántica el orden racional y predecible de la geometría neoclásica de los jardines de Versalles fue sustituido por un bosque tenebroso e inhóspito lleno de rayos, truenos, y peligros en el caos, la complejidad y lo impredecible ${ }^{31}$.

Los románticos construyeron un sistema dinámico basado en la idea de que la naturaleza está regida por fuerzas espirituales y que la materia es el resultado de fuerzas opuestas. En el fondo la reacción de Goethe es ya una profunda rebelión contra la visión fragmentada de las cosas, que se fue imponiendo en la profesionalización de la ciencia durante el siglo XVIII, y que heredaba una determinada racionalidad desde la Ilustración ${ }^{32}$. El esquema newtoniano de difracción de la luz para generar los colores del espectro visible era para Goethe una simplificación de un fenómeno que habla que estudiar desde un punto de vista físico, químico, fisiológico, artístico o emocional. En el mundo romántico, quizás recuperando algunas ideas de la vieja filosofla natural o anticipando algunas de las críticas de Snow, la ciencia había de formar parte de una sola cultura, y la comprensión de la naturaleza debía estar integrada de una manera holistica y orgánica más que

31 Cunningham, A., Jardine, N, (eds.) Romanticism and the Sciences. Cambridge University Press. Cambridge 1990; KNIGHT, David. La era de la Ciencia...op. cit.

32 Frangsmyr, Tore., HeIlbRON, John., RIDER, Robin E. (eds.) The Quantifying Spirit in the Eighteenth Century. University of California Press. Oxford 1990. 
mecánica, donde los colores eran al mismo tiempo luz, química, o emociones generadas en su contemplación ${ }^{33}$. De la misma forma, personajes de la talla de Alexander von Humboldt concebía sus exploraciones en tierras incógnitas tropicales como auténticos retos de descripción global de la naturaleza en todas sus manifestaciones, desde la medida de la presión y la temperatura en función de la altura en la ascensión de una cima de los Andes, hasta la descripción emocionada del paisaje contemplado por el explorador, aventurero y cientifico ${ }^{34}$.

El mundo romántico de las primeras décadas del siglo XIX integraba, por ejemplo, la electricidad como una de las manifestaciones de las polaridades y fuerzas ocultas de la naturaleza, y ese misterioso fluido podía explicar, por un lado, la famosa pila de Volta, o el gavanismo, la medicina eléctrica, o incluso restablecer la fuerza vital en un cadáver, tal como el Dr. Frankenstein planeaba para su criatura. Así, Big-bang, Caos, Gaia, Catástrofes, son quizás como una nueva revolución contra la simplificación mecánica que ya La Mettrie había propuesto en el siglo XVIII, estimulan nuestra inteligencia, que parece todavía algo más que un conjunto artificial de semiconductores y circuitos integrados que con la tecnologia informática desarrollan una llamada «inteligencia artificial" que un día nos podría llegar a desafiar, com el famoso HAL, sucedáneo de IBM, en el ya clásico film de Stanley Kubrick "2001 una odisea del espacio», o a desdibujar nuestro concepto de realidad a través de nuevas imágenes virtuales.

De hecho, la nueva "religión" de la tecnociencia de finales del siglo XX, a pesar de conseguir progresos innegables parece tener notables grietas. En la tradición positivista de August Comte y el pensamiento evolucionista de finales del siglo XIX, la ciencia se ha abierto camino triunfante sobre otras formas de pensamiento como la magia, la religión o la misma filosofía. Hoy muy pocos comparten este optimismo científico. Aunque la mayoría valora la capacidad que ha tenido la ciencia moderna de liberarnos de esas otras tradiciones para construir una metodología y un modelo propio, la actitud del usuario de cajas negras de nuestro mundo tecnológico donde se genera una confianza implícita y muy poco razonada hacia la máquina, quizás no están demasiado lejos del pensamiento mágico de sociedades primitivas.

33 BurWICK, F., The Damnation of Newton: Goethe's Color theory and romantic Perception. de Gruyter, Berlin and New York, 1986.

34 BơtTING, Douglas, Humboldt. Un savant Démocratique. Belin. Paria 1988 (1a ed. en inglés 1973). 
En realidad, uno de los grandes dilemas en nuestra sociedad tecnocientifica es el de la difusión de esos mismos valores culturales a la población en general. Sin ciencia y tecnología será difícil sobrevivir en el siglo XXI, pero las materias tradicionales consolidadas como disciplinas académicas durante el siglo XIX parecen claramente obsoletas e inadecuadas para explicar los vertiginosos cambios que se han producido en los últimos decenios. Además, la crisis de las humanidades tradicionales no parece ayudar a la construcción de los anhelados puentes de Snow entre las dos culturas. ¿Qué valores y qué tipo de formación instrumental requieren los usuarios de las múltiples cajas negras que acompanan nuestra vida cotidiana? E incluso, ¿qué tipo de información científica se debe transmitir al público en general, a los ciudadanos anónimos de los países industrializados en el siglo XXI? La respuesta a estas preguntas no parece sencilla, pero la ignorancia de la propia historia de la ciencia y de la tecnología, asi com la negligencia de sus consiguientes bifurcaciones continuas y coexistencias de modelos y estilos de diversos, encorsetan todavía más nuestra visión actual del problema. Los científicoshistoriadores del siglo XIX construyeron las historias míticas de los grandes héroes de la ciencia, que habían forjado las nuevas profesiones de científicos profesionales, pero olvidaron la importancia de las continuas competencias tecnológicas entre múltiples máquinas e inventos, la notable contribución a la cultura cientifica de las figuras de segunda fila, o la reiterada persistencia de debates sobre las fronteras entre la ciencia y la pseudociencia, o entre la ciencia y la religión en diversos momentos históricos. Sólo una revisión en profundidad de nuestra cultura tecnocientifica enriquecida desde su dimensión histórica puede contribuir a encontrar las claves educativas que permitan una mejor comprensión y utilización de las nuevas tecnologías de la información o de la vida.

A pesar de que las últimas aproximaciones constructivistas de la propia ciencia y de la tecnología han sido y continúan siendo controvertidas, probablemente aportan elementos importantes de cara a la introducción en nuevos proyectos educativos (com el famoso Ciencia-Técnica-Sociedad). Así, deberíamos hablar de la importancia de las prácticas científicas en espacios concretos; o insistir en la importancia del estudio de los objetos, de los instrumentos, de un tácito «savoir faire", de los espacios de validación, y de estandarización y control de la propia actividad científica ${ }^{35}$. Desde el llamado "Strong programme"

35 PESTRE, Dominique, «Pour une histoire sociale et culturelle des sciences. Nouvelles définitions, nouveaux objets, nouvelles pratiques", Annales Histoire, Sciences Sociales, mai-juin 1995, págs. 487-522. 
o programa fuerte de la Escuela de Edimburgo, de David Bloor y Barry Barnes, hasta las teorfas más radicales del «actor-network» de Michel Callon o de Bruno Latour, en los últimos años ha emergido un amplio abanico de escuelas y de enfoques diversos que ponen especial énfasis en la construcción social del mismo conocimiento científico, el cual, ahora ya no disfruta de aquel estatus privilegiado, neutral y notablemente independiente de los factores culturales o sociales que se le otorgaban hace sólo unas décadas ${ }^{36}$. Así las disciplinas científicas y las estrategias de "seducción" de audiencias sustituyen a los tradicionales discursos presentistas sobre la profesionalización o institucionalización de la ciencia; los espacios de práctica científica, en especial el laboratorio, se priorizan en relación a las grandes ideas o teorías; los discursos retóricos y la proyección pública que acompaña a menudo la ciencia, así como sus instrumentos y objetos, y su representación visual, son presentados como prioritarios en relación a una visión más tradicional de experimentos, instrumentos y enseñanza científica como meras estrategias mecánicas de corroboración de las grandes teorías ya establecidas. Por último, las particularidades, las contingencias y los contextos locales toman una importancia mayor, así como las redes de validación que ellos mismos constituyen. El mismo Dominique Pestre, junto con John Kriege, ambos editores de un importante libro colectivo sobre la historia de la ciencia en el siglo XX, presentan en su introducción más de diez definiciones diferentes de la ciencia contemporánea, que, a su vez, condicionan nuestra aproximación histórica a la misma: ${ }^{37}$ Una manera de buscar la verdad a través de la racionalidad; un divertimento, un placer que satisface la curiosidad personal; una moral social y política que ayuda a construir una sociedad más objetiva y justa; una batalla contra la ignorancia, la superstición y la religión; la actividad de unos expertos financiados por el estado o por el ejército; un monstruo capaz de transgredir las reglas de la naturaleza; un sistema de instituciones educativas y de investigación; una práctica inaccesible y casi mágica para los profanos; un recurso retórico, un lenguaje que confiere prestigio y autoridad; un buen medio de ascensión social; una colección de instrumentos y máquinas.

Desde estas perspectivas, la ciencia y la tecnología representan mucho más que los descubrimientos e inventos de las grandes figuras, y se acercan al problema de los valores y prácticas concretas. La ciencia puede ser así entendida

36 GOLINSKI, Jan, Making Natural Knowledge...op. cit., págs. 13-46.

37 KRIGE, John and PESTRE, Dominique, "Introduction" a KRIGE, John and PESTRE, Dominique (eds.) Science in the Twentieth Century. Harwood. Amsterdam 1997, págs. xxii-xxv. 
como una estrategia social para difundir determinados valores, para contribuir al reforzamiento del orden social, o para crear un nuevo lenguaje de comunicación en una red de practicantes. Un nueva historia más simétrica ${ }^{38}$, capaz de tomar en consideración experimentos socialmente aceptados así como experimentos ignorados puede contribuir a relativizar el peso de los grandes nombres y la frecuente imagen de recepción pasiva de los científicos locales en relación a ellos ${ }^{39}$, así como evitar típicas discusiones sobre grandes genios, atraso científico, pesimismo o optimismo radical.

\section{Conclusión}

Algunos de los ejemplos anteriores han sido escogidos entre muchos otros posibles para señalar algunas de las cuestiones importantes que se plantean en nuestra cultura científica actual. Quizás Levi-Straus tenia razón y la consistencia de esta cientificidad condiciona ya todos nuestros actos. En cualquier caso, me parece saludable apuntar la posibilidad de que algunas piezas del rompecabezas no encajen bien. El problema más difícil es quizás ver el cuadro general con objetividad suficiente para analizar la ciencia desde fuera de sí misma. Probablemente dentro de cada ser humano hay una curiosidad innata por saber cómo somos y de donde vienen las cosas que nos rodean y las conexiones neuronales de su cerebro se extienden de manera radial y misteriosa para producir genialidades como la relatividad einsteniana o el sueño de estructuras moleculares, como en el caso del químico alemán August Kekulé. No debemos, sin embargo olvidar que, en el futuro, el desfile supuestamente triunfal de los ejércitos de la tecnociencia moderna está amenazado por la protesta de nostálgicos filósofos naturales disfrazados de Frankenstein. El reto consiste en hacerles frente de manera constructiva y original, evitando un torpe enfrentamiento cara a cara, o una sumisión acrítica a su poder. Y en el fondo, se trata evidentemente de un gran reto educativo.

38 BLOOR, David, Knowledge and social imagery. London, 1976, BARNES, B. SHAPIN, S. (eds.), Natural order: historical studies of scientific culture. London, 1979.

39 Para la historia de la tecnología, ver: W.E. BIJKER, T.P. HuGHES, and T.J. PINCH (eds.), The Social Construction of Technological Systems. New Directions in the Sociology and History of Technology MIT Press, Cambridge, Mass 1987. 